\title{
La Real Cittadella di Messina Approccio architettonico alle preesistenze e restauro Antonio Bonifacio
}

Scuola di Architettura di Siracusa - SDS dell’Università di Catania, Siracusa, Italia, arch.bonifacio@gmail.com

\begin{abstract}
Researches have offered many new contributions, both documentaries (about Carlos de Grunenbergh's formation and his architectural formulations related to the cultural reference models, and about the long phase of construction of the Royal Citadel, including the used materials and techniques) and, above all, about the physical consistency of the surviving structures, taking into consideration the detected stratifications as well.

The results of these studies have been object of several educational experiences of experimentation design, since the knowledge and the historical-critical understanding of such a subject can highlight the guidelines and the appropriated instructions to approach and develop the restoration, interpretating the conservative instances coming from the work itself. The very interesting, topical subject about the quality of a project, seen from the perspective of an architectural approach to the pre-existences, is based on its comprehension itself and requires a deep relationship between conservation and innovation.
\end{abstract}

Keywords: restauración, conservación, innovación, reutilización.

\section{Introduzione}

La Real Cittadella di Messina è una fortificazione "alla moderna", voluta dal Re di Spagna come emblema della potenza e memoria della repressione, dopo la riconquista successiva alla ribellione antispagnola del 1674-1678. Fu progettata e realizzata, nel decennio seguente al 1680, dall'ingegnere militare Carlos de Grunenbergh, che la impiantò alla radice della penisola falcata, origine e significato della città stessa, porta d'ingresso per la Sicilia e a diretto contatto con l'abitato, il porto e lo Stretto di Messina.

L'impianto stellare originario, pentagonale con opere esterne, è stato implementato, nel corso del XVIII secolo, con una lunetta, varie batterie ed opere secondarie. Ulteriori le stratificazioni del XIX e fino al sisma del 1908, che provocò seri danni, ma lasciò sostanzialmente integre le opere originarie. Nel Novecento, ciò che la natura aveva risparmiato, è stato parzialmente e progressivamente demolito, sfigurato ed abbandonato fino al punto che, oggi, rimangono solo alcune parti in stato di rovina.

\section{Carlos de Grunenbergh}

Sono numerosi ed autorevoli gli studi sulla produzione grafica, sui progetti e sulle realizzazioni di Carlos de Grunenbergh, in particolar modo quelli sulle opere siciliane realizzate tra il 1671 ed il 1687, senza tralasciare l'attività prestata a Malta e di nuovo in Sicilia nella ricostruzione dopo il terremoto del 1693.

Storia e storiografia ci restituiscono la figura di un ingegnere militare, di origine fiamminga, al servizio della corona spagnola, che operò principalmente in Spagna, insieme col fratello Fernando, e poi in Sicilia. Entrambi i fratelli, militari di formazione, accreditati di grande 
esperienza nella pianificazione di difese e fortificazioni, nonché di opere di ingegneria idraulica, si sarebbero formati nelle Fiandre, loro regione di provenienza. I loro modelli culturali di riferimento, pertanto, in relazione ai sistemi difensivi ed alle formulazioni architettoniche dagli stessi proposte, sono stati fino ad oggi ritenuti olandesi e fiamminghi per origine e formazione, spagnoli per servizio prestato, francesi per informazione indiretta dovuta a servizi di intelligence militare. ${ }^{1}$

Il riscontro di nuovi materiali d'archivio ci impone una riflessione sulla figura di Carlos de Grunenbergh ed un ripensamento sulle sue formulazioni architettoniche estremamente originali ed innovative.

Nato a Colonia ${ }^{2}$ da nobile famiglia originaria della Vestfalia, ${ }^{3}$ raggiunse giovanissimo Parigi per studiare, ${ }^{4}$ ed è ancora in Francia quando, tra i tumulti locali, giura fedeltà al Re di Spagna per difendere l'onore del suo $\mathrm{Re}$ e dell'Aquila Austriaca. ${ }^{5}$ La prima notizia relativa a Carlos de Grunenbergh in Spagna è del 1656 "ingeniero, este hombre tiene inteligencia en la profesión aunque no ha praticado en la guerra", ${ }^{6}$ dove giunge col titolo di "Coronel de Alemanes". ${ }^{7} \mathrm{Da}$ altri documenti si evince che lo stesso fece richiesta di essere restituito ai tedeschi, quando fu incarcerato perché reo, con altri, di avere lasciato ai francesi il Forte del SS. Salvatore di Messina, nel 1676, durante la rivolta antispagnola. $^{8}$

Carlos de Grunenbergh era, dunque, tedesco, colonnello in quanto nobile, ingegnere formatosi, probabilmente, in Francia e con poca esperienza dal punto di vista strettamente militare all'epoca del suo arrivo in Spagna. Fu, poi, particolarmente attivo in Galizia, ove, col fratello, nel corso dell'ultima fase della guerra di restaurazione portoghese, tra il 1662 ed il 1666, ne migliorò il sistema difensivo, restaurando, ricostruendo, ampliando ed implementando le fortezze esistenti. ${ }^{9}$

I de Grunenbergh, nel 1667-68, presero parte alla "guerra di devoluzione" contro la Francia di Luigi XIV e, rientrati a Madrid, presentarono alla reggente Marianna d'Austria un Memorial nel quale proposero la realizzazione di un canale laterale per rendere navigabile il Manzanarre, il Jarama ed il Tago a Toledo. ${ }^{10}$ Nel 1669 giunsero in Italia nello Stato dei Presidii toscani e nel regno di Napoli. Successivamente, nell'estate del 1671, Carlos de Grunenbergh giunse in Sicilia dove, per volontà del viceré Claude Lamoral, principe di Ligne, ebbe il compito di visionare il sistema difensivo della costa orientale, ritenuta la più debole e che necessitava di attenzione immediata, al fine di provvedere al suo miglioramento ed implementazione.

Le opere di Carlos de Grunenbergh in Sicilia sono perfettamente documentate e conosciute attraverso le fonti dell'Archivo General de Simancas di Valladolid. ${ }^{11}$ Dopo la costruzione della Torre de Ligny a Trapani del 1671, a seguito di sopralluoghi condotti nel $1673 \mathrm{col}$ viceré principe di Ligne, propose soluzioni per migliorare le difese di Catania, Augusta e Siracusa. Come risulta evidente dai documenti, il sistema di fortificazione proposto dal de Grunenbergh si basava su linee avanzate davanti ai fronti esistenti delle città, come avvenne ad Augusta e Siracusa. Tanto nei restauri delle fortificazioni più antiche, quanto nella proposizione di quelle nuove, la difesa venne migliorata con la moltiplicazione di opere esterne quali rivellini, mezzelune, opere a corno. Soluzioni già presenti nei disegni di Francesco de Marchi, ${ }^{12}$ ma senz'altro rivoluzionarie in quanto, per la prima volta, si tentava di tenere il nemico a distanza attraverso la realizzazione di opere esterne, con l'intento di prolungare l'assedio.

Questo sistema di fortificazione si sviluppò in ambito francese a partire dalle teorie di Pagan ${ }^{13}$ quale evoluzione del cosiddetto "sistema olandese" di Marolois ${ }^{14}$ e, successivamente, grazie alle realizzazioni del Vauban. ${ }^{15}$ Questi ebbe il grande merito di usare e moltiplicare le opere avanzate di primo fronte, proprio come fece Carlos de Grunenbergh nei suoi lavori in Sicilia durante gli stessi anni, influenzando i progetti ideati dagli ingegneri militari di tutta Europa alla fine del XVII secolo e per tutto il XVIII.

Le opere di Carlos de Grunenbergh mostrano chiaramente, dunque, i riflessi della scuola 
francese nell'architettura militare, per l'uso, la diffusione e, principalmente, per la concreta realizzazione delle opere esterne.

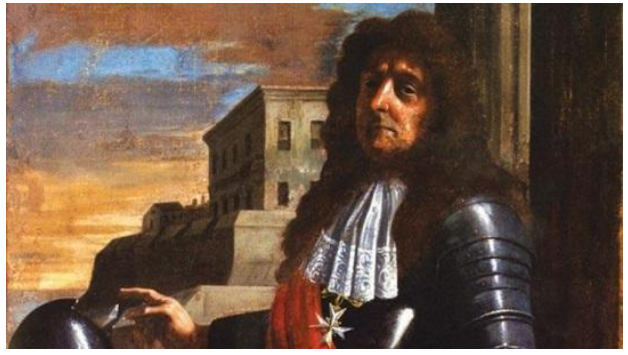

Fig. 1 - Mattia Preti (attribuito), Don Carlos de Grunenbergh (dettaglio), Malta, 1687, collezione privata. Olio su tela, cm 130 x 165 .

\section{La Real Cittadella di Messina}

Le cittadelle bastionate erano considerate, soprattutto, degli strumenti di controllo e di repressione e la loro costruzione rientrava pienamente nella logica del potere assoluto, che non ammetteva ribellioni da parte della popolazione. Queste, insieme con le altre fortificazioni, erano l'espressione della tirannia degli Asburgo ed il modello universale di potere non solo in Sicilia, ma in tutti i territori della monarchia spagnola. Per questi motivi, riconquistata la città dopo i moti del 1674-78, il viceré Francisco de Benavides y Dávila, Conte di Santo Stefano, decise di affidare la realizzazione ex novo della fortificazione a Carlos de Grunenbergh, la cui fama, per il numero e la qualità delle sue formulazioni, oltre che l'ampiezza delle sue conoscenze, garantivano il soddisfacimento delle esigenze del potere.

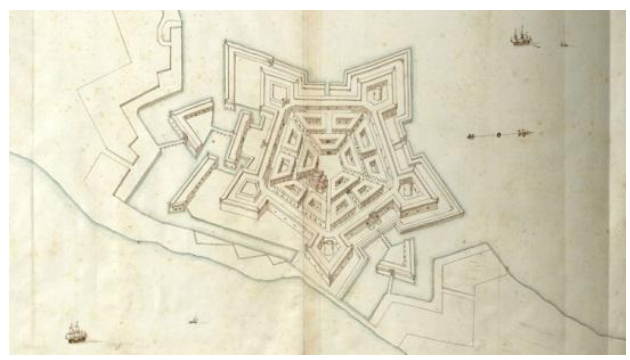

Fig. 2 - Carlos de Grunenbergh (attribuito), Ciudadela de Mecina, Palermo 1686 (si veda la nota $16 / d)$.
Il modello proposto e realizzato alla radice della penisola falcata, a partire dal 22 aprile 1680 , mostra ancora una volta tutta la perizia dell'ingegnere militare, non solo per il tracciato innovativo ed all'avanguardia dal punto di vista prettamente fortificatorio, ma anche per le soluzioni tecniche e tecnologiche adoperate per la realizzazione dell'opera stessa, la cui complessità derivava principalmente dal doverla fondare sul mare.

Una piazza d'armi pentagonale con gli acquartieramenti, realizzati poi solo parzialmente rispetto al progetto finale cui fanno riferimento i diversi disegni del $1686,{ }^{16}$ posti all'interno del recinto primario con cortine e bastioni, una falsa braca parallela sulla cui prosecuzione, in elevato, verso Terranova, furono posizionate due controguardie davanti ai baluardi a formare una tenaglia. Tra di loro un opera rettilinea davanti alla cortina e, a conclusione, un rivellino. Altro rivellino fu posizionato verso San Raineri, dove le previste controguardie non furono realizzate perché ritenute non necessarie, data la presenza, da quella parte, del Forte S. Salvatore e della Lanterna. Tutto intorno alla falsa braca erano i fossati allagati. I due bastioni verso Terranova erano pieni con cavaliere poligonale superiore, gli altri tre, vuoti, con cavaliere cilindrico posto nello spazio interno con funzioni di polveriera. Le gallerie delle cortine avevano funzioni di ricovero di munizioni eccetto quella tra $\mathrm{i}$ baluardi di S. Stefano e S. Diego che conteneva quattro cisterne.

I tre baluardi vuoti ed i loro cavalieri, come tutte le cortine, erano voltati all'interno e lastricati nella parte superiore, dove, sui parapetti esterni, erano posizionate le troniere.

Altra grande innovazione di Carlos de Grunenbergh fu quella di posizionare nei vani interni voltati delle opere esterne, quali rivellini, avanti cortina, e controguardie, oltre che nei cavalieri dei baluardi pieni, tutti quanti lastricati e con le troniere sui parapetti esterni, le casematte per il tiro corto.

Sul parapetto della falsa braca coronata, con piattaforma e terrapieno, erano posizionate le 
troniere. Nel rivellino della Grazia, verso S. Raineri, erano i forni ed i depositi per la farina.

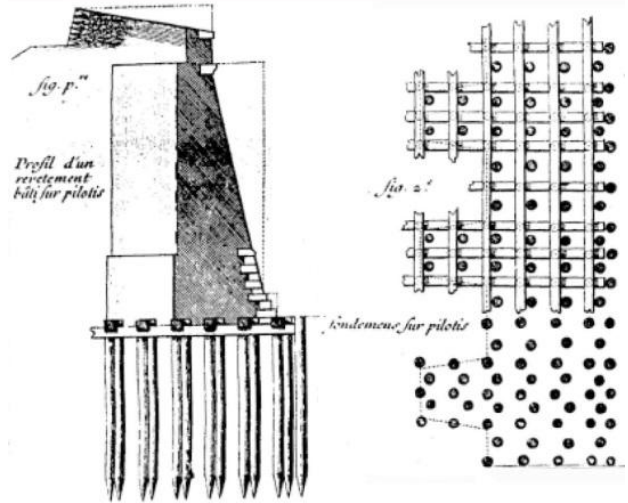

Fig. 3 - Sistema di fondazione su pali secondo Bernat Forest de Bélidor, in La science des ingenieurs..., Parigi, 1729, L. III, Pl. 8, p. 64.

Ancora più sorprendenti le tecnologie adoperate per le opere marittime. I fossati allagati, tanto profondi da potere essere navigabili dal lato del porto, erano comunicanti con quelli più interni realizzati sotterraneamente tra la piazza d'armi e le cortine della falsa braca. Questi canali voltati, in comunicazione col mare per mantenere le acque pulite col moto continuo e col riflusso, formavano delle piccole darsene coperte dalle quali era possibile soccorrere con barche $\mathrm{i}$ rivellini o disturbare il nemico che cercasse di attraversare i fossati principali. ${ }^{17}$

Quanto alle tecniche costruttive, nuovi, importantissimi, apporti ci vengono da fonti d'archivio. ${ }^{18}$ Il complesso sistema di fondazione fu congegnato su "palaccioni", pali di legno che venivano infissi nel terreno e nel mare con l'ausilio di machinae a percussione. I legni, provenienti dai boschi della Calabria e di Collabascia, nel territorio di Castiglione di Sicilia, avevano differenti sezioni e venivano utilizzati a seconda della qualità del terreno (quelli a sezione maggiore venivano usati per il terreno meno solido). I pali, nella porzione non incassata nel terreno, venivano inglobati in uno strato di pozzolana, superiormente al quale si realizzava un piano in tavolato ligneo o in pietra.

Lo scavo dei fossati fu fatto con l'ausilio di molini con trombe calafatate a pece per aggottare l'acqua. Lo stesso sistema fu utilizzato per lavorare a secco nell'infissione dei pali e la successiva elevazione delle fabbriche.

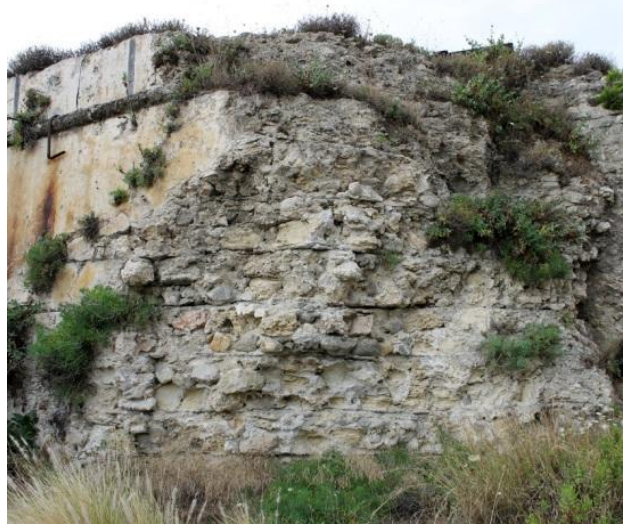

Fig. 4 - Dettaglio della sezione muraria della cortina-fronte 3 .

Le imponenti sezioni murarie furono realizzate con cortine in "pietra viva" e massicciate interne con puddinghe a cemento prevalentemente calcareo, costipate per battitura in malta di calce idraulica. Le demolizioni effettuate nel Novecento ci consentono, oggi, di riconoscere chiaramente, all'interno delle sezioni murarie, tanto i livellamenti realizzati con regolarità, quanto questa sorta di concrezione, la cui eccezionale tenacità ha garantito il comportamento monolitico, in cui si distinguono il legante reso idraulico, gli elementi lapidei (provenienti soprattutto dalla demolizioni di fabbriche preesistenti) e le cortine murarie. La stessa tecnica costruttiva venne usata per le strutture voltate. Gli archi di volta in "pietra viva" perfettamente connessi con le cortine con la soprastante massicciata. I cantoni "moligni" furono realizzati prevalentemente con calcari provenienti da cave locali, quali quelle di Gravitelli o di Cammari, ${ }^{19}$ quelle di San $\mathrm{Leo}^{20} \mathrm{e}$ di Bauso. ${ }^{21}$ Sempre in pietra di San Leo fu realizzato il cordone di tutte le fabbriche, mentre le troniere, le porte, le finestre e quasi tutte le opere in cui era necessaria una maggiore lavorazione furono realizzate in pietra di Siracusa, più tenera del calcare "a polipai". Altro litoide utilizzato è la pietra "misca" di Trapani. ${ }^{22}$ 
Tutte le superfici architettoniche, infine, furono protette con intonaci a base di calce idraulica.

L'impianto originario fu successivamente implementato con ulteriori opere esterne. Alcuni trinceramenti vennero realizzati dagli spagnoli intorno al 1719 , sotto assedio austriaco. Una di queste traverse, sulla linea della controguardia di S. Stefano e verso lo Stretto, aveva un fossato con cavallo di frisia dalla parte di Terranova. Su questa ed altra traversa parallela, poco più a Nord, venne installata dai Borbonici, tra il 1735 ed il 1753, una batteria con terrapieno detta di San Francesco. Nel 1770 fu costruita, tra la stessa controguardia ed il rivellino di S. Teresa, la lunetta Carolina, intitolata così in onore di Maria Carolina d'Asburgo-Lorena, arciduchessa d'Austria e moglie di Ferdinando III re di Sicilia. Poco tempo dopo, la lunetta Carolina fu unita alla batteria di S. Francesco che divenne, così, la lunetta di S. Francesco. Più avanti, alla fine del XIX secolo, quando dalla parte del porto erano già iniziate le demolizioni, nella batteria di S. Francesco venne svuotato il terrapieno e trasformata in cisterna, da cui l'attuale definizione di "Cisternone". Tutte queste fasi possono ancora oggi essere lette e verificate con una lettura stratigrafica degli elevati.

Le demolizioni iniziate a fine Ottocento per la realizzazione di un passaggio verso S. Raineri, consistite nel taglio parziale della controguardia di S. Carlo e di parte della falsa braca dalla parte del porto, sono proseguite, poi, nel Novecento per la realizzazione della stazione marittima con la definitiva e progressiva demolizione di tutte le fabbriche di quella porzione di fortezza. Dopo la seconda guerra mondiale, cedute le aree ai cantieri navali, altre opere sono state demolite, al punto che, oggi, rimangono solo alcune parti in stato di rovina.

Le fabbriche superstiti, ad una lettura del quadro fessurativo, mostrano la presenza di dissesti statici manifestatisi in tempi relativamente lunghi ed originati da cedimenti fondali indotti dal sisma del 1908. I moti traslatori hanno innescato rotazioni reciproche tra le parti, causando profonde lesioni passanti con andamento lineare e longitudinale sulle chiavi degli archi di volta.

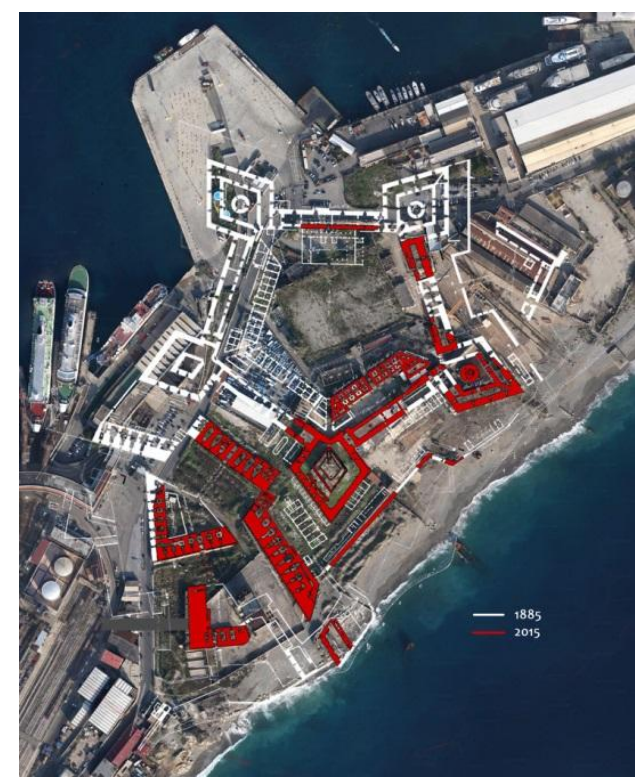

Fig. 5 - Strutture superstiti (elaborazione grafica ESEMeP 2014)

Gli intonaci originari, a larghi tratti lacunosi, sottoposti alla prolungata esposizione agli agenti atmosferici, si mostrano sovente rigonfi, distaccati o esfoliati, complessivamente o tra $\mathrm{i}$ vari strati che li compongono.

I materiali lapidei naturali sopra descritti, tanto per le loro caratteristiche chimico-fisiche, quanto per l'azione degli agenti atmosferici, presentano degradazioni quali erosione, alveolizzazione, disgregazione. Le malte dei solidi murari, non più protette, si presentano spesso polverizzate. Ingenti le mancanze di porzioni murarie.

\section{Approccio alle preesistenze e restauro}

Gli esiti di questi studi sono stati posti alla base di varie esperienze didattiche di sperimentazione progettuale, $^{23}$ nella consapevolezza che la conoscenza e la comprensione storico-critica dell'oggetto debbano mettere in evidenza, con l'interpretazione delle istanze conservative provenienti dall'opera stessa, i criteri guida e le corrette indicazioni di metodo per l'approccio progettuale al restauro. Operazione, questa, da intendersi come attività scientifica con fondamento filologico, volta a rivelare, conservare ed evidenziare le preesistenze, 
assicurando una lettura chiara e storicamente corretta.

Il tema, di grande attualità, della qualità del progetto, nell'approccio architettonico alle preesistenze, è fondato sulla suddetta comprensione storico-critica, trovando il proprio presupposto nell'esigenza di un'intima relazione tra conservazione ed innovazione. In questa sperimentazione progettuale, ove hanno parte prioritaria quelle operazioni di carattere strettamente tecnico tese a preservare dal degrado la consistenza fisica e materiale delle opere, tutte le formulazioni proposte con le modalità proprie del linguaggio architettonico dovranno trovare una chiara, coerente e rigorosa relazione concettuale con le preesistenze. Col restauro si deve tendere ad assicurare la conservazione e l'uso dell'opera, tenendo, però, ben presente che la sola operazione di attribuzione di nuova funzione ad un manufatto architettonico, anche se a carattere sociale, non rappresenti l'obiettivo primario dell'intervento.

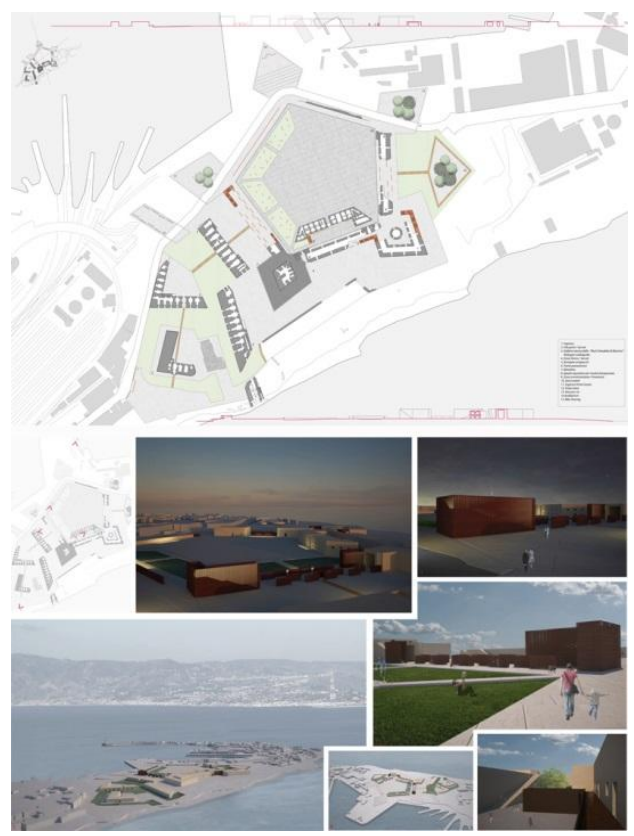

Fig. 6 - Progetto Altomare, Arrogante, Bruno, Belfiore, Coccimiglio, Fucile, Greco, Incognito.

Le esperienze didattiche condotte, finalizzate all'interpretazione delle istanze conservative provenienti dalle opere stesse, hanno consentito di chiarire ulteriormente che la Real Cittadella di Messina costituisce un patrimonio culturale che può (ancora) essere assoggettato ad interventi di restauro volti a ritrovare, conservare ed evidenziare le preesistenze, per garantirne la conservazione, la trasmissione al futuro e l'uso. Senza dimenticare, infine, che i messinesi attendono di riappropriarsi, con la zona falcata, della loro origine, della loro storia e del significato della loro città.

\section{Note}

(1) Cfr. Boscarino S., Catania: le fortificazioni alla fine del Seicento ed il piano di ricostruzione dopo il terremoto del 1693, in "Quaderni dell' I.D.A.U di Catania", n. 8, Catania-Caltanissetta, Vito Cavallotto Editore, 1976, pp. 69-102.

(2) "Sortì la culla in Colonia Agrippina Città...." Polizzi G., Oratione funebre nella morte del Colonnello sig. D. Carlo de Grunembergh Caualiero di deuotione, ed ingegniero maggiore per S.C.M. in questo Regno di Sicilia. Composta dal Reuerendo Padre Don Geronimo Polizzi chierico regolare..., Messina, per Matteo La Rocca, 1696, p. 7.

(3) “... frà le palme gloriose della Famiglia de' Conti di Vestfalia”. Ivi, p. 8.

(4) “...e corre à Parigi, imbiancando con quei Gigli le stole dell'Innocenza, infiora il suo intelletto con le più vaghe Primauere di Pallade." Ivi, p. 9 .

(5) "E Carlo à guisa del Sole pria attrahe a se i vapori, e poi à suo tempo qual nembo si squarcia in folgori, qual turbine scoppia in tuoni, custodit atrium suum, non insanguina la spada, se non, per difendere del suo Re l'honore, e Carlo così giura fedeltà al Monarca Ibero, che pria martire dell'honore seppeliscesi in Francia entro catomba d'horrori, che fatto condottiere d'armati con tempeste di fuoco mouer guerra contro l'Aquila Austriaca." Ivi, p. 13.

(6) AGS (Archivo General de Simancas, Valladolid), Guerra y Marinas, leg. 1879, citato in Siracusa, città e fortificazioni. Catalogo della mostra dal medesimo titolo (Siracusa 1987), a cura di Dufour L., Palermo, Sellerio, 1987, p. 69, nota 206. 
(7) AGS, Negociado de Guerra, leg. 1985 e 2163, citato in Siracusa, città e fortificazioni, op. cit., p. 50.

(8) Don Josepe Albion Calascibetta così scriveva: “. . y dar orden para la prision de los referidos perseguidos sin admitir la pretencion del coronel Don Carlos Grunembergh de ser remitido a los alemanes siendo absurda y contra la forma de las odenanzas militares cuyo traslado va adjunto, respecto de que el coronel no vino a esse reyno con regimientos Alemanes si no en tiempo de paz como ingenieros y se hallò despues con los cabos militares españoles en el castillo del Salvador en el qual no havia ningun aleman ..." AGS, Estado-Sicilia, leg. 3524-20, citato in: Manfrè V., Imágenes urbanas y coleccionismo geográfico en la Sicilia de Edad Moderna. Tesis doctoral, Madrid, Universidad Autónoma, 2014, vol. 2, p. 103.

(9) Cfr. Manfrè V., op. cit., vol. I, p. 285-291.

(10) Cfr. De Grunenbergh C., De Grunenbergh F., Memorial que los coroneles, Don Carlos, y Don Fernando de Grunenbergh han dado à su Magestad, tocante à la proposicion que tenian hecha, derendir nauegable à Mançanares desde la otra parte del Pardo hasta Toledo ..., 1668.

(11) Cfr. Aricò N., Carlos de Grunenbergh e le città ioniche del Teatro geografico antiguo $\mathrm{y}$ moderno del reyno de Sicilia (1686), in "Lexicon", n. 7, Palermo, Caracol, 2008, pp. 2336.

(12) Di Francesco de Marchi (1504-1576), si veda: Della architettura militare, del capitanio Francesco de' Marchi ... Libri tre. Nelli quali si descriuono li veri modi, del fortificare, che si vsa a' tempi moderni. Con vn breue, et vtile trattato, nel quale si dimostrano li modi del fabricar l'Artigliaria, e la prattica di adoperarla, da quelli che hanno carico di essa, Brescia, appresso Comino Presegni ad instanza di Gasparo dall'Oglio, 1599.

(13) Blaise François Pagan, conte di Merveilles (1603-1665). Per prolungare la difesa vengono duplicati i recinti, moltiplicate le artiglierie, migliorata la difesa dei fossi. Il fronte bastionato, inoltre, deve adattarsi al terreno. $\mathrm{Si}$ veda: Pagan B. F., Les fortifications du comte de Pagan, Paris, chez Cardin Besogne, 1645.

(14) Samuel Marolois (1572-1627). Propone i bastioni perpendicolari alla cortina, introduce una falsa braca alla quota del cammino coperto con la stessa figura del recinto principale e posiziona un semplice rivellino, le cui gole sono sull'allineamento della controscarpa, davanti alle cortine. Si veda: Marolois S., Fortification ou Architecture militaire tant offensive que defensive; supputée et dessignée par Samuel Marolois, Haga Comitis, ex officina Henr. Hondii, 1615.

(15) Di Sébastien Le Prestre de Vauban (16331707), si veda: Traité de l'attaque et de la défense des places. Par M. le maréchal de Vauban, La Haye, chez Pierre De Hondt, 17421743.

(16) I disegni sono: (a) Carlos de Grunenbergh, Planta de la ciudadela, ciudad y Puerto de Messina, 20 de junio de 1686, AGS, MPD XI29. (b) Carlos de Grunenbergh, Planta de la ciudadela, ciudad y Puerto de Messina, 31 de julio de 1686, AGS, MPD XI-30. (c) Carlos de Grunenbergh (attr.), Mecina, in Teatro geografico antiguo y moderno del Reyno de Sicilia, 1686, Palermo, 1 de mayo 1686, BMAEC (Ministerio de Asuntos Exteriores y de Cooperación, Archivo General y Biblioteca, Madrid), Ms. 3, f. 17. (d) Carlos de Grunenbergh (attr.), Ciudadela de Mecina, in Teatro geografico..., BMAEC, Ms. 3, f. 18.

(17) Cfr. (a) AGS, Estado-Sicilia, leg. 3504-134. Relacion del estado en que se halla la presente la fábrica de la nueva Ciudadela. (Don Rodrigo Godinez Brochero y Texada, Don Ignacio Gaston). Citato in: Manfrè V., op. cit., vol. 2, p. 94 e ss. (b) FBM (Fundación Bartolomè March, Palma de Mallorca), ms. B81-F-12. Relación del Gobierno de Sicilia, del Conde de Santisteban, desde 11 de Diciembre de 1678 que tomó posesión del, hasta el día 11 de junio 1687 haviendole sucedido el Duque de Uzeda. Citato in: Manfrè V., op. cit., vol. 2, p. 164 e ss.

(18) Cfr. Relaciones y certificaciones del dinero gastado en la fábrica de la ciudadela de Messina y en la fortificaciones de la ciudades de 
Messina y Agosta en los virreinatos del conde de San Esteban y el duque de Uceda. SNAHN (Sección Nobleza del Archivo Histórico Nacional, Toledo), FRIAS, C.75, D.27-32.

(19) Calcare biancastro massivo, brecciato, polverulento e vacuolare del messiniano, a cui si affiancano marne, sabbie e calcareniti plioceniche. Definizione da: Lentini F., Catalano S., Carbone S., Carta Geologica della Provincia di Messina, Firenze, S.EL.CA., 2000.

(20) Calcare a polipai bianco e grigio, di natura sedimentaria, con setti a corallo visibili sino in superficie (minerale di base $\mathrm{CaCO} 3$ ). Definizione da: Lentini F., op. cit. La dizione di San Leo, fa riferimento alle cave di contrada Tremonti, nel quartiere San Leone.

(21) Calcare siliceo compatto, talvolta a struttura brecciata, di colore tra l'avorio ed il rosa carico tendente al rossastro. Definizione da: Lentini F., op. cit.

(22) Frammenti calcarei con elementi anfibolitici nella struttura, di colore grigio con l'aggiunta di macchie biancastre/rossastre. La pietra misca (o mischio) di Trapani, definita nei documenti come "misco della banca", fu fortemente voluta dal de Grunenbergh in quanto proveniente dal banco roccioso su cui egli stesso, nel 1671, aveva costruito la Torre de Ligny a Trapani. Più tardi questo litoide fu impiegato da Luigi Vanvitelli per la scala monumentale della Reggia di Caserta.

(23) (a) Universidad de Sevilla, Politechnika Lubelska, Università degli Studi Mediterranea di Reggio Calabria, EMDiReB - European Master in Diagnosis and Repair of Buildings, Corso 2012-14, III semestre specialistico, Reggio Calabria. (b) Scuola di Architettura di Siracusa, SDS dell'Università di Catania, CLM in Architettura, Corso di Restauro, a.a. 2013-14 e 2014-15. (c) ESEMeP - Ente Scuola Edile di Messina, Corso per Tecnico per la conservazione ed il riuso dei beni culturali, 2014.

\section{References}

Aricò N. (2008). "Carlos de Grunenbergh e le città ioniche del Teatro geografico antiguo y Aricò N., (2008) "Carlos de Grunenbergh e le città ioniche del Teatro geografico antiguo y moderno del reyno de Sicilia (1686) in Lexicon. n. 7. Palermo. Caracol. pp. 23-36.

Boscarino S. (1976). "Catania: le fortificazioni alla fine del Seicento ed il piano di ricostruzione dopo il terremoto del 1693" in Quaderni dell I.D.A.U di Catania. n. 8. Catania-Caltanissetta. Vito Cavallotto Editore. 1976. pp. 69-102.

De Grunenbergh C., De Grunenbergh F. (1668). Memorial que los coroneles, Don Carlos, y Don Fernando de Grunenbergh han dado à su Magestad, tocante à la proposicion que tenian hecha, derendir nauegable à Mançanares desde la otra parte del Pardo hasta Toledo .... (manoscritto).

Ioli Gigante A. (1978). "La costruzione della cittadella di Messina attraverso alcune carte dell'Archivio generale di Simancas (Valladolid)" in Archivio storico messinese. III s., XXIX . pp. 45-58.

Ioli Gigante A. (1980). Messina. Roma-Bari. Laterza. (collana "Le città nella storia d'Italia").

Manfrè V. (2014). Imágenes urbanas y coleccionismo geográfico en la Sicilia de Edad Moderna. Tesis doctoral. Madrid. Universidad Autónoma.

Penisola (la) di San Raineri. (2002). Diaspora dell'origine. a cura di N. Aricò. "Rassegna di studi e ricerche". n. 4 D.R.P - Dipartimento di Rappresentazione e Progetto dell'Università di Messina. Messina. Sicania. 2002.

Polizzi G. (1696). Oratione funebre nella morte del Colonnello sig. D. Carlo de Grunembergh Caualiero di deuotione, ed ingegniero maggiore per S.C.M. in questo Regno di Sicilia. Composta dal Reuerendo Padre Don Geronimo Polizzi chierico regolare... Messina. per Matteo La Rocca.

Riccobono F., Berdar A., La Fauci C. (1988). La Real Cittadella di Messina. Messina. EDAS.

Siracusa, città e fortificazioni. (1987). Catalogo della mostra dal medesimo titolo (Siracusa 1987). a cura di Dufour L. Palermo. Sellerio.

Vilardo R. (2004). "La Real Cittadella di Messina" in Quadrato. n. 2. D.R.P - Dipartimento di Rappresentazione e Progetto dell'Università di Messina. Messina. Sicania. 